\title{
Primary prophylaxis of gastric variceal bleeding: the choices need to be tested!!
}

\author{
Chitranshu Vashishtha ${ }^{1} \cdot$ Shiv Kumar Sarin ${ }^{1}[$
}

Received: 16 April 2021 / Accepted: 23 June 2021 / Published online: 10 August 2021

(c) Asian Pacific Association for the Study of the Liver 2021

Gastric varices $(\mathrm{GV})$ pose a challenging problem in hepatology; due to the risk of severe variceal bleeding as well recurrent hepatic encephalopathy related to co-existent large spleno-renal shunts. Primary GV (varices seen in initial endoscopic examination, before any therapy) are seen in $17 \%$ of patients of liver cirrhosis, the prevalence being higher in bleeders than non-bleeders; $24 \%$ vs $7 \%$. Secondary GV appear for the first time after therapy for esophageal varices (EV), and are seen in $9 \%$ of patients with portal hypertension [1]. The 'Sarin Classification' is universally applied to determine the natural history, choice of therapeutic intervention and likely outcomes in patient with GV [2]. According to it, GV are divided into two subgroups, the gastroesophageal varices (GOV) and isolated gastric varices (IGV). GOV are further subclassified as GOV1 when esophageal varices extend below the gastroesophageal junction along lesser curvature of stomach and as GOV2 when the esophageal varices extend into the fundus of stomach. IGV includes IGV1, when located in fundus, and IGV2, when located elsewhere in the stomach [2]. GOV1 constitute nearly $75 \%$, GOV2-21\%, IGV1 less than $2 \%$, and IGV2 about $4 \%$ of all GV. The location of GV is important in predicting the frequency of bleed (IGV1 $>$ GOV2 $>$ GOV1). The GOV1 are in fact continuation of esophageal varices and the dominant afferent portal venous feeder is the left gastric vein originating from distal splenic vein, proximal part of main portal vein or splenoportal confluence. The hemodynamics and the management of GOV1 are similar to that of EV, i.e., endoscopic band ligation. For the same reason, bleeding from these veins has a favorable response to transjugular intrahepatic porto-systemic shunt (TIPS). For IGV1 and GOV2 collaterals, the dominant afferents are the

Shiv Kumar Sarin

shivsarin@gmail.com; sksarin@ilbs.in

1 Department of Hepatology and Liver Transplant, Institute of Liver and Biliary Sciences, New Delhi 110070, India posterior (64-70\%) and short (25-64\%) gastric veins arising from the proximal portion of splenic vein [3].

In about $10 \%$ patients with portal hypertension, spontaneous portosystemic shunts develop to accommodate the hyperdynamic and large blood volume in the portal circulation. The frequency of such shunts increases to about $85 \%$ in patients with GV. Predominantly these are left sided shunts, i.e., to the left of midline and include gastrorenal shunts (GRS), direct gastro-caval shunts and gastrocaval shunts via inferior phrenic vein. More than $90 \%$ of these shunts are GRS.

Acute variceal bleed is a dreaded complication of portal hypertension with $20 \%$ mortality risk at 6 weeks in cirrhosis [4]. GV attribute about $20 \%$ of all variceal bleeds, but they are associated with more risk of uncontrolled bleeding, higher transfusion requirements, rebleeding and death compared to EV. At present, the available treatment options for large ( $>10 \mathrm{~mm})$ and high risk $\mathrm{GV}(>20 \mathrm{~mm}$, with red color signs), without history of prior bleed, are obliteration by cyanoacrylate glue, occlusion through the spontaneous porto-systemic shunts or transjugular intrahepatic portosystemic shunt (TIPS) (Fig. 1). Use of cyanoacrylate glue is the treatment of choice for the control of acute GV bleed and for the prevention of rebleed from GV. Glue therapy offers a success rate close to $95 \%$ in the control of acute bleed and nearly $92 \%$ in preventing rebleed [5]. Only a very small proportion of patients with GV bleed fail to endotherapy, especially those with portal vein thrombosis, severe coagulopathy or with incomplete obturation [6].

Hepatic venous pressure gradient (HVPG) is the most important method to predict the risk of esophageal variceal bleeding, success of endotherapy and rebleeding. HVPG, however, does not correlate well with the risk of bleeding from $\mathrm{GV}$ and is not much different between bleeders and non-bleeders [7]. In fact, GV can bleed at HVPG less than $12 \mathrm{~mm} \mathrm{Hg}$, while the threshold of continued bleeding as well as early recurrence of bleeding from esophageal varices is considered to be $16 \mathrm{mmHg}$ [8]. In $\mathrm{GV}$, the risk of bleed 
Fig. 1 Anatomy of the portosystemic shunts, afferents, efferents and various treatment options. The white arrowheads denote the usual afferents of the gastric varices and black arrowheads denote the efferent pathway through gastrorenal shunt. BRTO balloon-occluded retrograde transvenous obliteration; $G V$ gastric varices; $I V C$ inferior venacava; $L G V$ left gastric vein; $L R V$ left renal vein; $P G V$ posterior gastric vein; $S G V$ short gastric vein; $S M V$ superior mesenteric vein; $S V$ splenic vein; TIPS transjugular intrahepatic portosystemic shunt (may be combined with variceal embolotherapy)

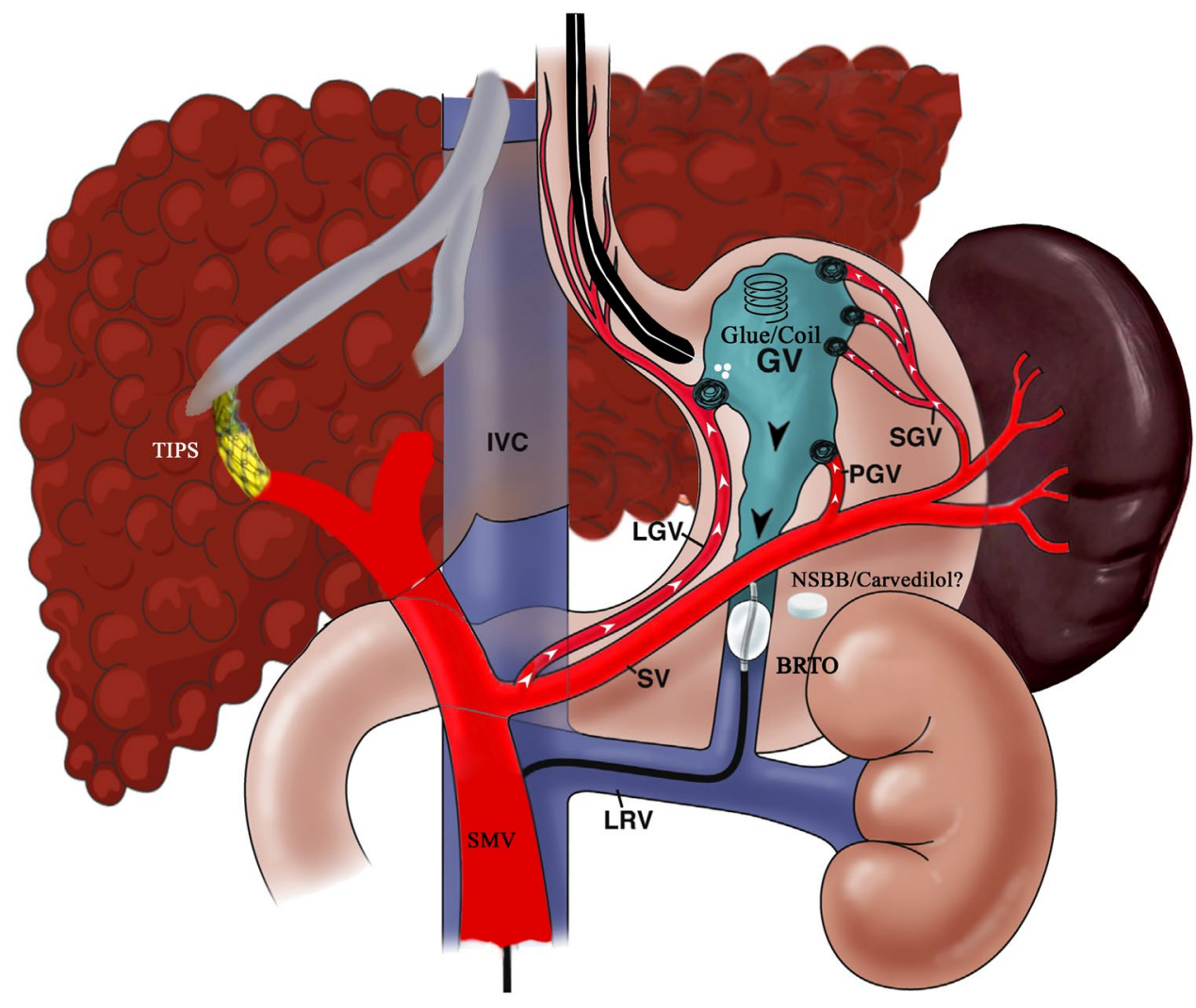

depends on the size of varices ( $>2 \mathrm{~cm}$ ), wall tension, presence of red color signs on the varices, MELD $\geq 17$ and presence of portal hypertensive gastropathy [7, 9].

Due to lack of correlation with HVPG, TIPS is not considered a very effective intervention in the control of acute GV bleed. In fact, in the absence of a large gastro-renal shunt, TIPS with afferent embolization should be preferred over TIPS alone [10]. The role of prophylactic TIPS in the prevention of first GV bleeding is another contentious area. Neither prophylactic surgical shunts, nor TIPS, have gained popularity in patients with large GV. Non-selective betablockers (NSBBs) are the mainstay for primary prophylaxis of esophageal varices. Whether substantial reduction of HVPG occurs by beta-blockers, and they can be of help in prevention of GV bleed, has not been well studied. In one study, propranolol was found to be superior to no-therapy, but inferior to glue therapy, in the prevention of first variceal bleed [7]. A recently published double-blind placebo-controlled RCT (PREDESCI) concluded that in compensated cirrhotics, NSBBs prevent not only variceal bleeding, but also other types of decompensation, including ascites [11]. However, patients of GV were not adequately represented in this study. Reduction in portal pressure is likely to reduce the frequency of decompensation episodes. Carvedilol, which is more potent than propranolol should be evaluated to achieve greater reduction in portal flow and decrease in the incidence of first bleed from GV.
Balloon-occluded retrograde transvenous obliteration (BRTO) procedure utilizes the GRS to access the GV. The GRS is occluded with a balloon and sclerosant is injected in the GV. This causes damage to the endothelial lining and complete occlusion or reduction of the vascular bed. Hence, BRTO is especially useful in GOV2/IGV1 with GRS. A recently published randomized controlled trial has demonstrated its superiority in preventing rebleed from gastric varices compared to cyanoacrylate glue injection [12]. However, BRTO needs hours of monitoring as occlusion balloon needs to stay long enough to obliterate GV [13]. Hence, modified BRTO techniques have been developed. These are PARTO (using vascular plugs), CARTO (using coils and gel foam slurry) and BATO (accessing GV antegradely when GRS is absent, through percutaneous transhepatic route or trans TIPS). PARTO utilizes a permanent vascular plug to reduce post-procedural monitoring time as well as avoiding risk of balloon rupture. It also eliminates the need of sclerosant, thereby avoiding its complications, such as hemolysis, renal failure and disseminated intravascular coagulation. CARTO is specifically useful in the presence of large shunts (upto 25-30 $\mathrm{mm}$ in diameter), difficult angle or tortuous vessel prohibiting the use of BRTO or PARTO [14]. Endoscopic ultrasound (EUS) has shown promise in the management of GV. It can differentiate GV from prominent gastric folds and guide obliteration of GV by glue and/or coil under real-time imaging, confirming the procedural success. Other 
modalities with sparse data are endoscopic thrombin injection and hemospray [15].

Compared to esophageal varices, there are little data on the primary prophylaxis and treatment of GV bleed. There is only one randomized controlled study in patients of cirrhosis for primary prophylaxis of GV bleed. Mishra et al. studied 89 patients of cirrhosis with large high-risk GOV2 and IGV1 for primary prophylaxis of GV bleed. Patients were randomized into three groups: cyanoacrylate glue injection until complete obliteration (group I), or propranolol with a target heart rate of 55/min or maximal dose of $360 \mathrm{mg} /$ day (group II), or no treatment (group III). Subsequently, they were followed for a median of 26 months. The actuarial probability of bleeding from $\mathrm{GV}$ was $13 \%$ in group I compared to $28 \%$ in group II $(p=0.039)$, and $45 \%$ in group III $(p=0.003)$. The actuarial probability of survival was higher in group I compared to group III ( $90 \%$ vs $72 \%, p=0.048)$. However, there was no significant difference in mortality observed between group I vs group II [7].

In the current issue of Hepatology International, Choe et al. contribute an important work on primary prophylaxis of GV bleed. In this retrospective study from Korea, 210 patients of cirrhosis of liver with GV were analyzed for primary prophylaxis of GV bleed. Seventy-two underwent endoscopic variceal obturation (EVO) using n-butyl cyanoacrylate glue diluted with ethiodized oil, 41 underwent BRTO and 97 were observed for a median follow-up of 35 months. Patients with significant EV were excluded. Primary end-point of the study was GV bleeding rate which was seen significantly more in the observation group (35.1\%) than in EVO (19.4\%) and BRTO group (7.3\%). BRTO group had a tendency for lower rate of GV bleed compared to EVO group, but was not significant statistically. However, complete GV eradication rate was higher in BRTO than EVO group. Importantly, subgroup analyses showed that patients with concomitant GRS had a higher GV bleed rate in the EVO group (23.5\%) than BRTO group (7.3\%) $(p=0.032)$. These data indicate that in patients who have not bled from $\mathrm{GV}$, and have a large GRS, BRTO may lower bleeding risk, though with no survival benefits. These data need to be confirmed in a well-designed prospective RCT [16].

Another interesting observation in the study by Choe et al. was that even if the patients receiving $\beta$ blockers were excluded, the analysis and conclusions were unchanged. Similarly, the probability of bleeding in the three groups remained unchanged when analyzing both the esophageal and gastric variceal bleed. An important limitation of the study is the lack of baseline HVPG data. This would have helped in determining the complications of BRTO, which have been reported to be very low. BRTO in fact increases the portal pressure by upto $44 \%$ from the baseline and can lead to development of ascites or bleeding from esophageal varices [17]. Another important issue in the Korean study is that the all-cause mortality, a harder point, did not improve in the intervention groups than the observation group. Accordingly, neither glue nor BRTO are likely to reduce mortality from preventing first bleed from GV. These data are quite at variance with the clinical observations and prior studies. There is need to undertake large prospective studies in this cohort of patients.

A recent meta-analysis compared BRTO with TIPS in patients of cirrhosis with GV bleed; 353 patients from five clinical controlled studies were analyzed. No significant difference was observed between the two methods for technical success, hemostasis and complication rates. However, rebleed and hepatic encephalopathy were more in TIPS [18]. Overall, BRTO and TIPS should be considered as complimentary in the management of patients with GV with bleed. TIPS can be helpful in simultaneous control of ascites and EV bleed risk, but predisposes the patients to encephalopathy. BRTO can be helpful in simultaneous control of encephalopathy and bleeding, but can worsen ascites and pre-existing esophageal varices. We suggest an algorithm for primary prophylaxis of bleeding from gastric varices (Fig. 2).

Overall, Choe et al. should be lauded for their efforts and their work should serve as an impetus for future prospective randomized controlled studies on primary prophylaxis of gastric variceal bleeding. 


\section{Suggested Algorithm for Primary prophylaxis of Gastric Variceal Bleed}

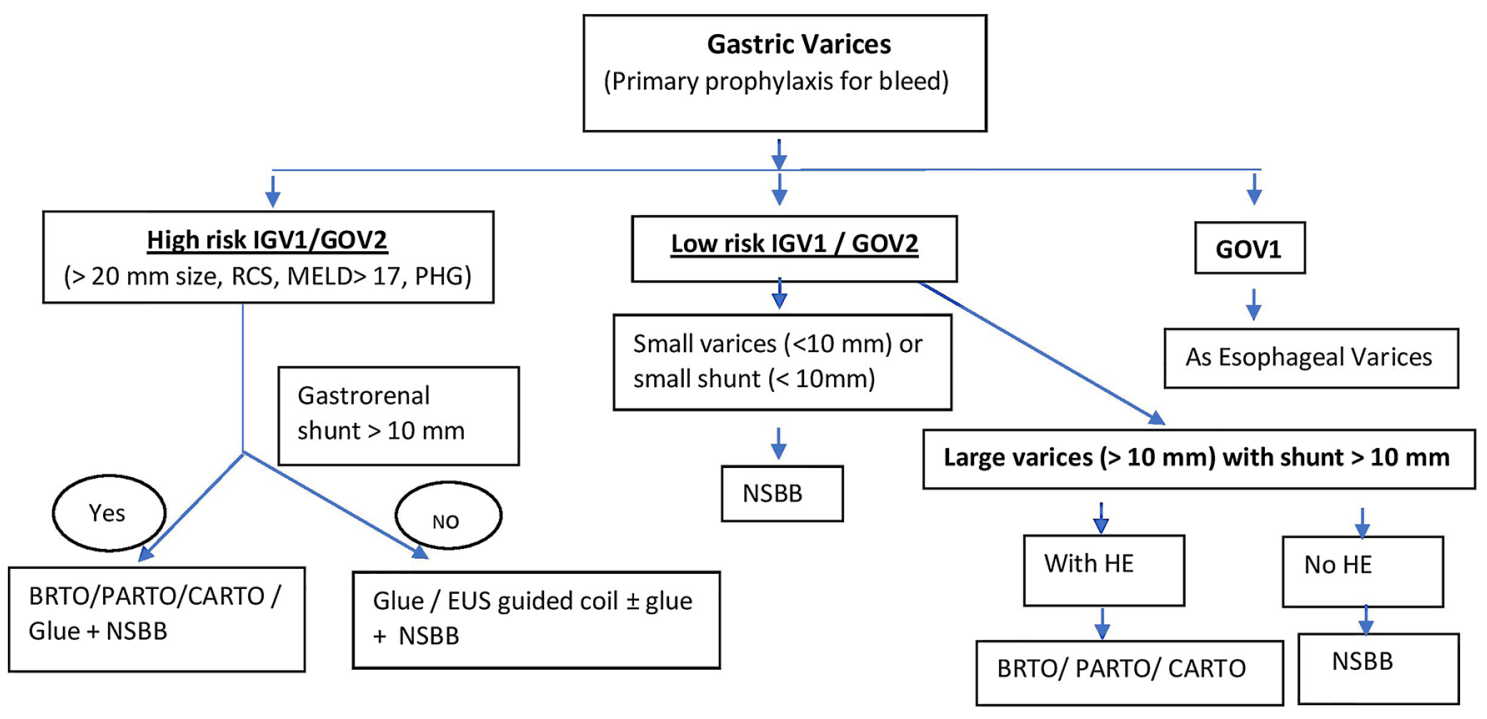

Fig. 2 Suggested algorithm for primary prophylaxis of gastric variceal bleed. BRTO balloon-occluded retrograde transvenous obliteration; PARTO plug-assisted retrograde transvenous obliteration;

\section{Declarations}

Conflict of interest The authors declare that they have no conflict of interest.

\section{References}

1. Sarin SK, Lahoti D, Saxena SP, et al. Prevalence, classification and natural history of gastric varices: a long-term follow-up study in 568 portal hypertension patients. Hepatology. 1992;16:1343-1349

2. Sarin SK, Kumar A. Gastric varices: profile, classification and management. Am J Gastroenterol. 1989;84:1244-1249

3. Chikamori F, Kuniyoshi N, Shibuya S, Takase Y. Correlation between endoscopic and angiographic findings in patients with esophageal and isolated gastric varices. Dig Surg. 2001;18(03):176-181

4. Ardevol A, Ibanez-Sanz G, Profitos J, et al. Survival of patients with cirrhosis and acute peptic ulcer bleeding compared with variceal bleeding using current first line therapies. Hepatology. 2018;67:1458-1471

5. Al-Ali J, Pawlowska M, Coss A. Endoscopic management of gastric variceal bleeding with cyanoacrylate glue injection: safety and efficacy in a Canadian population. Can J Gastroenterol. 2010;24:593-596

6. Kind R, Guglielmi A, Rodella L, Lombardo F, Catalano F, Ruzzenente A, et al. Bucrylate treatment of bleeding gastric varices: 12 years' experience. Endoscopy. 2000;32:512-519

7. Mishra SR, Sharma BC, Kumar A, Sarin SK. Primary prophylaxis of gastric variceal bleeding comparing cyanoacrylate injection and beta-blockers: a randomized controlled trial. J Hepatol. 2011;54:1161-1167
CARTO coil-assisted retrograde transvenous obliteration; NSBB nonselective beta blockers; $E U S$ endoscopic ultrasonography; $R C S$ red color signs; $P H G$ portal hypertensive gastropathy

8. Ready JB, Robertson AD, Rector W Jr. Effects of Vasopressin on portal pressure during hemorrhage from esophageal varices. Gastroenterology. 1991;100:1411-1416

9. Kim T, Shijo H, Kokawa H, Tokumitsu H, Kubara K, Ota K, Akiyoshi N, Iida T, Yokoyama M, Okumura M. Risk factors for hemorrhage from gastric fundal varices. Hepatology. 1997;25:307-312

10. Saad W. Endovascular management of gastric varices. Clin Liver Dis. 2014;18:829-851

11. Villanueva C, Albillos A, Genesca J, et al. Beta blockers to prevent decompensation of cirrhosis in patients with clinically significant portal hypertension (PREDESCI): a randomised, double-blind, placebo-controlled, multicentre trial. Lancet. 2019;393:1597-1608

12. Luo X, Xiang T, Wu J, Wang X, Zhu Y, Xi X, et al. Endoscopic cyanoacrylate injection vs BRTO for prevention of gastric variceal bleeding: a randomised controlled trial. Hepatology. 2021. https:// doi.org/10.1002/HEP.31718

13. Mukund A, Rajesh S, Arora A, Patidar Y, Jain D, Sarin SK. Efficacy of balloon-occluded retrograde transvenous obliteration of large spontaneous lienorenal shunt in patients with severe recurrent hepatic encephalopathy with foam sclerotherapy: initial experience. J Vasc Intervent Radiol. 2012;23:1200-1206

14. Kim DJ, Darcy MD, Mani NB. Modified balloon-occluded retrograde transvenous obliteration (BRTO) techniques for the treatment of gastric varices: vascular plug-assisted retrograde transvenous obliteration (PARTO)/ coil-assisted retrograde transvenous obliteration (CARTO)/balloon-occluded antegrade transvenous obliteration (BATO). Cardiovasc Intervent Radiol. 2018:41:835-847

15. Philips CA, Ahamed R, Rajesh S, George T, Mohanan M, Augustine P. Beyond the scope and the glue: update on evaluation and management of gastric varices. BMC Gastroenterol. 2020;20:361

16. Choe JW, Yim HJ, Lee SH, Chung HH, Lee YS, Kim SY, et al. Primary prophylaxis of gastric variceal bleeding: endoscopic 
obturation, radiologic intervention, or observation? Hepatol Int. 2021. https://doi.org/10.1007/s12072-021-10154-1

17. Uehara $\mathrm{H}$, Akahoshi $\mathrm{T}$, Tomikawa $\mathrm{M}$, et al. Prediction of improved liver function after balloon-occluded retrograde transvenous obliteration: Relation to hepatic vein pressure gradient. J Gastroenterol Hepatol. 2012;27:137-144

18. Wang YB, Zhang JY, Gong JP, Zhang F, Zhao Y. Balloonoccluded retrograde transvenous obliteration versus transjugular intrahepatic portosystemic shunt for treatment of gastric varices due to portal hypertension: a meta-analysis. J Gastroenterol Hepatol. 2016;31:727-733

Publisher's Note Springer Nature remains neutral with regard to jurisdictional claims in published maps and institutional affiliations. 